\title{
Advancing the Frontier of Hydraulics Experimentation using Ferrofluids
}

\author{
Laura Maria STANCANELLI \\ Delft University of Technology TU-Delft, Faculty of Civil Engineering and Geosciences, \\ Delft, The Netherlands \\ e-mail: L.M.Stancanelli@tudelft.nl
}

\begin{abstract}
Ferrofluids (FFs) are colloid liquids susceptible to magnetic field. Although they represent a novelty in Hydraulics, they have been widely used in engineering sciences since the eighties, with different industrial and biomedical applications. Here, a collection of experiences carried out in hydraulic laboratories is presented. Indeed, FFs have been exploited to measure key hydraulic quantities (e.g. wall friction). Moreover, their interaction with flow have been investigated with original techniques (optical methods, microfluidics) observing the rise of novel phenomena (e.g. lubrication).
\end{abstract}

Keywords: ferrofluids, wall shear stress, lubrication, PTV, microfluidics.

\section{INTRODUCTION}

Ferrofluids (FFs) are formed by magnetic nanoparticles coated with a surfactant (preventing agglomeration) and dispersed in a liquid carrier (water or oil). One of their most important characteristics is their susceptibility to the magnetic field. FFs are very flexible due to: i) their tunable rheology; ii) the possibility of controlling their shape through their responsiveness to a magnetic field; iii) the fluid state, whereby the particles are free to move, showing lubricant properties; iv) the capability to hold the FF in place using an externally applied magnetic field.

The dynamics of ferrofluids have been extensively studied since the seventies (Shliomis 1972; Patel et al. 2013). Because of these peculiar characteristics, ferrofluids have been widely used in different applications (i.e. loudspeakers, bearings, micro valves, drug targeting, cancer therapy). In the following, a series of laboratory experiments carried out with ferrofluids are described. In particular, it is shown how ferrofluids can be exploited to measure wall friction and how their interaction with the surrounding flow gives rise to novel phenomena (e.g. lubrication) that can be investigated with original techniques (optical methods, microfluidics). 


\section{EXPERIENCES WITH FERROFLUIDS IN HYDRAULIC LABS}

\subsection{The development of an innovative wall shear stress measurement technique}

The measurement technique takes advantage of the above described properties of ferrofluids to measure fluid-wall shear stresses, with possible applications on sandy bottoms (Stancanelli et al. 2020). The principle of operation is really simple (Musumeci et al. 2015a,b; 2018). A ferrofluid drop, $\mathrm{O}(1 \mathrm{~mm})$ high, is placed on the wall and exposed to a permanent magnetic field. Therefore, this drop takes a conical shape that deforms under the action of the flow impacting on it. The measure of shear stresses exerted by the flow is performed by evaluating the ferrofluid drop deformation (i.e. the drop apex displacement) acquired by a camera. The technique is capable to measure changes in flow resistance induced by either skin friction and form drag. Experimental results demonstrated the capability to measure small shear stress variations (less than $0.001 \mathrm{~N} / \mathrm{m}^{2}$ ), as those associated with the change in roughness of the bimodal mixture composing the sediment bed.

\subsection{Ferrofluid-flow interaction investigated with PTV and in microfluidics}

Pressure-driven channel-flow experiments (square cross section $1 \mathrm{~cm} \times 1 \mathrm{~cm}$ ) have been carried out for investigating the ferrofluid-flow interaction. A stable ferrofluid layer is placed on one of the confining walls. This layer interacts with the fluid flowing within the channel, leading to a slip boundary condition. The ferrofluid layer is shaped and stabilized at the wall using an array of permanent magnets. Different steady flow regimes, characterized by Re numbers in the laminar, transitional and turbulent regime, have been tested. The flow field has been investigated by means of optical techniques (i.e., PIV, PTV). Flow velocity profiles and flow patterns within the channel have been extracted and compared with the flow regime observed in the absence of ferrofluid. The comparison demonstrates significant drag reduction $(>60 \%)$ in both laminar and turbulent regimes. Moreover, in order to further understand ferrofluid-flow interactions, as well as ferrofluids rheology, a series of experiments has been carried out at the micron scale. Microfluidic chips, such as straight channel (rectangular section $1500 \mu \mathrm{m} \times 110 \mu \mathrm{m}$ ), and Y-junction channels (rectangular section $1100 \mu \mathrm{m} \times 50 \mu \mathrm{m}$ ), have been used for this aim. In these specific settings, it was possible to study the reorganization of the ferrofluid structure under the action of different magnetic fields (i.e. chains formation composed of magnetite particles) and the phenomena acting at the interface of the ferrofluid and a co-flowing fluid (a mineral oil).

Acknowledgments. The work on the measurement technique has been funded by the EU project HYDRALAB PLUS (proposal number 64110) and was carried out at the University of Catania under the supervision of Prof. Rosaria Musumeci and Prof. Enrico Foti. The work on the analysis of ferrofluid-flow interaction has received funding from the European Union's Horizon 2020 research and innovation programme under the Maria Skłodowska-Curie grant agreement No. 841259 hosted at Swiss Federal Institute for Forest, Snow, and Landscape Research (WSL). The experimental research activity has been carried out at the ETH Zurich under the supervision of Prof Markus Holzner and in close collaboration with Eleonora Secchi. The author wish to thank members of the GIC group (UNICT) and of the EFM group (WSL, Birmendorf), and Prof Stefano Lanzoni (University of Padua) for the fruitful discussions, the useful comments and suggestions. 


\section{References}

Musumeci, R.E., V. Marletta, B. Andò, S. Baglio, and E. Foti (2015a), Ferrofluid measurements of bottom velocities and shear stresses, J. Hydrodyn. 27, 1, 150-158, DOI: 10.1016/S10016058(15)60467-X.

Musumeci, R.E., V. Marletta, B. Andò, S. Baglio, and E. Foti (2015b), Measurement of wave near-bed velocity and bottom shear stress by ferrofluids, IEEE Trans. Instrum. Meas. 64, 5, 1224-1231, DOI: 10.1109/TIM.2014.2359521.

Musumeci, R.E., V. Marletta, A. Sanchez-Arcilla, and E. Foti (2018), A ferrofluid-based sensor to measure bottom shear stresses under currents and waves, J. Hydraul. Res. 56, 5, 630-647, DOI: 10.1080/00221686.2017.1397779.

Patel, R., R.V. Upadhyay, and R.V. Mehta (2003), Viscosity measurements of a ferrofluid: comparison with various hydrodynamic equations, J. Colloid Interface Sci. 263, 2, 661-664, DOI: 10.1016/ S0021-9797(03)00325-4.

Shliomis, M.I. (1972), Effective viscosity of magnetic suspensions, Sov. J. Exp. Theor. Phys. 34, 6, 1291-1294.

Stancanelli, L.M., R.E. Musumeci, M. Stagnitti, and E. Foti (2020), Optical measurements of bottom shear stresses by means of ferrofluids, Exp. Fluids 61, 52, DOI: 10.1007/s00348-020-2890-3.

Received 22 March 2021

Accepted 12 April 2021 